\title{
The health and well-being of men who have sex with men (MSM) in Britain: Evidence from the third National Survey of Sexual Attitudes and Lifestyles (Natsal-3)
}

Catherine H. Mercer ${ }^{1 *}$, Philip Prah ${ }^{1}$, Nigel Field ${ }^{1}$, Clare Tanton ${ }^{1}$, Wendy Macdowall ${ }^{2}$, Soazig Clifton', Gwenda Hughes ${ }^{3}$, Anthony Nardone ${ }^{3}$, Kaye Wellings ${ }^{2}$, Anne M. Johnson ${ }^{1}$ and Pam Sonnenberg ${ }^{1}$

\begin{abstract}
Background: To date, research on men who have sex with men (MSM) has largely focused on their sexual health needs and on men recruited from gay-orientated venues. National probability survey data provide a rare opportunity to examine the broader sociodemographic, behavioural, and health profiles of MSM, defined as men who reported $\geq 1$ male sexual partner(s) in the past 5 years, and thus regardless of their sexual identity, in comparison to men reporting sex exclusively with women (MSEW) during this time, and also the extent that health inequalities cluster.

Methods: Britain's third National Survey of Sexual Attitudes and Lifestyles (Natsal-3), a probability sample survey, interviewed 15,162 people aged 16-74 years (6,293 men) during 2010-2012 using computer-assisted personal-interviewing with a computer-assisted self-interview. We used multivariable regression to compare MSM relative to MSEW in their reporting of variables, individually and collectively, corresponding to three domains: physical, mental, and sexual health.

Results: Among all men, $2.6 \%(n=190)$ were defined as MSM, of whom $52.5 \%$ (95 \% Cl: 43.6 \%-61.2 \%) identified as gay. MSM were as likely as MSEW $(n=5,069)$ to perceive their health was 'bad'/'very bad', despite MSM being more likely to report a long-standing illness/disability/infirmity (adjusted odds ratio, AOR: 1.46, 95 \% Cl:1.02-2.09), treatment for depression/past year (2.75, 1.69-4.47), and substance use (e.g., recreational drug use/past year: 3.46, 2.22-5.40). MSM were more likely to report harmful sexual health behaviours, e.g., condomless sex with $\geq 2$ partners/past year (3.52, 2.13-5.83), and poor sexual health outcomes, including STI diagnosis/es (5.67, 2.67-12.04), poorer sexual function (2.28, 1.57-3.33), both past year, and ever-experience of attempted non-volitional sex (6.51, 4.22-10.06). MSM were also more likely than MSEW to report poor health behaviours and outcomes both within and across the three health domains considered. Of all MSM, $8.4 \%$ had experienced poor health outcomes in all three domains - physical, mental, and sexual health - in contrast to $1.5 \%$ of all MSEW.

Conclusions: MSM are disproportionately affected by a broad range of harmful health behaviours and poor health outcomes. Although often observed for a minority of MSM, many health inequalities were seen in combination such that policies and practices aimed at improving the health and well-being of MSM require a holistic approach, regardless of clinical specialty.
\end{abstract}

\footnotetext{
* Correspondence: c.mercer@ucl.ac.uk

${ }^{1}$ Research Department of Infection and Population Health, University College

London, 3rd floor Mortimer Market Centre, London, UK

Full list of author information is available at the end of the article
} 


\section{Background}

Men who have sex with men (MSM) bear a disproportionate burden of sexually transmitted infections (STIs), including HIV $[1,2]$. However, sexual health extends beyond STI/HIV, and includes sexual function and sexual violence [3]. Furthermore, good sexual health is increasingly recognised as a key component of general health and well-being [4], with health behaviours and outcomes often sharing common risk factors that can exacerbate one another [5]. Of note, data suggest that gay men are at increased risk of poor mental health [6] and substance use [7], which can increase their likelihood of experiencing poor sexual health outcomes, including STI/HIV transmission [5].

To date, much of the evidence regarding MSM is based on those who attend sexual health clinics [8], or convenience surveys targeting MSM identifying as gay and/or recruit from gay-orientated venues [9], neither of which are representative of the diverse population of MSM [10]. In contrast, Britain's National Surveys of Sexual Attitudes and Lifestyles (Natsal) use probability sampling so the data can be considered as broadly representative of the general population [11], and include MSM regardless of their sexual identity. In this paper we use data from the most recent Natsal, Natsal-3, to examine the sociodemographic profile and sexual, mental and physical health of MSM, comparing them to men who reported sex exclusively with women (MSEW). We explore the extent that harmful health behaviours and poor health outcomes are reported in combination, and thus cluster within and across health domains, to capture the extent that inequalities are experienced by MSM relative to MSEW.

\section{Methods}

In Natsal-3, households across Britain were selected using stratified probability sampling from which one eligible individual was selected at random and invited to participate. Unlike Natsal-2 [12], there was no geographical over-sampling, for example, of areas that have large gay populations [13]. Altogether, 15,162 men and women aged 16-74 years (6,293 men) were interviewed. The overall response rate was $57.7 \%$ (of all addresses known or estimated to be eligible) consistent with other major social surveys undertaken contemporaneously, and the cooperation rate was $65.8 \%$ (of all contacted addresses known to be eligible). Full details of the methodology have been published [11].

Participants completed a combination of a face-to-face computer-administered personal interview (CAPI) and a computer-assisted self-interview (CASI). Participants were routed into the CASI if they reported sex with an opposite-sex partner and/or reported any sexual experience with someone of the same-sex (both since age 13), or if they refused to answer this question. The CASI included many questions about sexual behaviour, including: "Have you had sex with a man involving genital contact? That is oral or anal sex or any other contact involving the genital area." Men who reported that they had were then asked with how many men they had had sex with in various timeframes. In this paper we define MSM as men who reported sex with at least one man in the five years prior to interview. Participants were asked similar questions about their opposite-sex partners which we used to identify men who reported sex exclusively with women (MSEW) in the same timeframe, the five years prior to interview.

The CASI asked about participants' sexual health including their experience of STI diagnosis and nonvolitional sex. We assessed sexual function with the Natsal-SF, a validated measure that considers problems with sexual response, sexual function in the relationship context, and self-appraisal of sex life, among those sexually-active (defined as reporting sex in the past year) [14]. The CASI also asked about use of sexual health services including HIV testing, sexual health clinic attendance, and whether participants had sought help/advice about their sex life and if so, from where/whom.

Most of the data on general health and well-being were collected face-to-face using showcards so that participants needed only to report a letter code. These included questions about alcohol consumption, and experience of a range of chronic conditions, considered here as reporting none, one, or more than one such condition to capture comorbidity [4]. Reporting receiving treatment for depression from a health professional in the past year was one such condition but we considered it separately in order to consider mental health as a separate domain. Participants were deemed to have had depressive symptoms in the past two weeks if they scored a total of at least three in response to the validated two-question Patient Health Questionnaire (PHQ-2) $[15,16]$, which was included in the CASI.

After the CASI, questions about STI/HIV risk perception were asked face-to-face using showcards with Likert scale response options, followed by standard demographic items regarding ethnicity, sexual identity, social class (as measured by the National Statistics Socio-Economic Classification [17]), and area-level deprivation (as measured by the Index of Multiple Deprivation [18]).

The Natsal-3 study was approved by the Oxfordshire Research Ethics Committee A (reference: 09/H0604/27). Participants provided oral informed consent for interviews.

\section{Statistical analysis}

We did all analyses using the complex survey functions of Stata (version 13) to incorporate the weighting, clustering, and stratification of Natsal-3. We calculated descriptive statistics to compare MSM with MSEW, presenting agestandardised estimates to account for differences in age 
distributions. We used ordinal and binary multivariable logistic regression to calculate adjusted odds ratios (AORs) to investigate how the reporting of harmful health behaviours and poor health outcomes differed for MSM relative to MSEW.

We then considered the extent that MSM and MSEW reported multiple harmful behaviours within two health domains: physical and sexual health by summing the number of specific behaviours reported (a total of four harmful physical behaviours and three harmful sexual behaviours (Table 1)). We then used proportional Venn diagrams to consider the extent of overlap across health domains as the proportion reporting harmful behaviours in both health domains. We used the same approach to examine the reporting of multiple poor health outcomes within and across three different health domains: physical, sexual, and mental health (Table 1).

While Natsal-3 collected data on sexual identity, we do not present estimates for MSM stratified according to their sexual identity due to a lack of statistical power to make meaningful comparisons, but for reference our tables include estimates for the subset of MSM who identified as gay, including adjusted odds ratios relative to MSEW. We then summarise the differences observed relative to comparing all MSM to MSEW at the end of the Results section.

We used an $\alpha$ of 0.05 in all analyses.

\section{Results}

Prevalence of MSM \& reported numbers of sexual partners Among men aged 16-74 years resident in Britain, $2.6 \%$ [95 \% CI: $2.1 \%-3.0 \%$ ] were defined as MSM, corresponding

Table 1 Harmful behaviours and poor health outcomes reported in Natsal-3 used to define health domains

\begin{tabular}{|c|c|}
\hline Harmful behaviours & Poor health outcomes \\
\hline \multicolumn{2}{|l|}{ Domain: Mental health } \\
\hline Not available in Natsal-3 & $\begin{array}{l}\text { - Received treatment for } \\
\text { depression from a health } \\
\text { professional, past year }\end{array}$ \\
\hline \multicolumn{2}{|l|}{ Domain: Physical health } \\
\hline $\begin{array}{l}\text { - Current smoker } \\
\text { - Usual alcohol consumption greater } \\
\text { than recommended levels of } 21 \text { units } \\
\text { per week [34] } \\
\text { - Usually drink >8 units of alcohol } \\
\text { on one occasion ('binge drinking' [34]) } \\
\text { at least weekly } \\
\text { - Any illicit drug use (including cannabis), } \\
\text { past year }\end{array}$ & $\begin{array}{l}\text { - Current health described } \\
\text { as 'bad' or 'very bad' } \\
\text { - Long-standing illness/ } \\
\text { disability/infirmity } \\
\text { - } 1+\text { chronic condition } \\
\text { (excluding depression) } \\
\text { - Unhealthy BMl } \\
\text { (BMI <18|>25) [35] }\end{array}$ \\
\hline \multicolumn{2}{|l|}{ Domain: Sexual health } \\
\hline $\begin{array}{l}\cdot \geq 2 \text { condomless-sex partners, past year } \\
\cdot \geq 10 \text { sex partners, past year } \\
\cdot \text { Non-use of condoms at first sex with } \\
\text { any of the three most recent partners, } \\
\text { past year }\end{array}$ & $\begin{array}{l}\text { - STI diagnosis/es, past year } \\
\text { - Distressed/worried about } \\
\text { sex life, past year } \\
\text { - Experienced attempted } \\
\text { non-volitional sex, ever }\end{array}$ \\
\hline
\end{tabular}

to an unweighted total of $190 \mathrm{MSM}$ in Natsal-3. Of these men, 107 [52.5 \%, $95 \%$ CI: $43.6 \%-61.2 \%$ ] identified as gay, 34 [18.6 \%, $95 \%$ CI: $12.8 \%-26.3 \%$ ] as bisexual, and 45 [28.4 \%, $95 \%$ CI: $20.8 \%-37.5 \%$ ] as heterosexual/ straight.

MSM reported larger numbers of sexual partners - of either gender - than MSEW in all timeframes studied (Table 2). In the five years prior to interview, the majority of MSEW (62.7\%) reported one partner only, while this number was reported by $15.6 \%$ of MSM, with $34.8 \%$ of MSM reporting at least ten partners during this time, as did $6.3 \%$ of MSEW.

Most MSM (69.9 \%) reported at least one female partner in their lifetime, with a median of 2 female partners in contrast to a median of 6 among MSEW. Female partners accounted for $22.9 \%$ of all partners reported by MSM. Among MSEW, 2.5 \% reported at least one male partner to date but, by definition, not in the past five years. Similar proportions of MSM and MSEW - one in five - reported at least one new female partner in the past year.

\section{Sociodemographic profiles}

MSM were slightly younger than MSEW (Table 3). Around $40 \%$ of MSM were neither living with a partner nor in a steady relationship in contrast to $17.8 \%$ of MSEW. MSM were more likely than MSEW to describe their ethnicity as white (96.8 \% vs. $88.4 \%$ ), and less likely to consider religion as fairly/very important (23.4\% vs. $33.8 \%)$. MSM had higher educational attainment than MSEW, with some evidence of higher socio-economic status, but no difference in area-level deprivation.

\section{Health behaviours and outcomes}

Alcohol consumption was similar between MSM and MSEW (Table 4), but MSM were more likely to report being a current smoker (aAOR:1.72), and any recreational drug use (past year, aAOR:3.46), in particular, using drugs other than cannabis, which was reported by one-quarter of all MSM. There was no difference between MSM and MSEW in their reporting of having ever injected non-prescribed drugs. A minority of MSM and MSEW described their health as 'bad'/'very bad'. Similarities also existed in the proportions reporting chronic health conditions, but MSM were more likely than MSEW to report that they had a long-standing illness, disability, or infirmity (aAOR:1.46), perceive they had health condition(s)/disability/ies that had affected their sexual activity/enjoyment (past year, aAOR:2.24), and report taking medications they perceived limited their sexual activity/enjoyment (past year, aAOR:2.56). The proportions screening positive for depressive symptoms using the PHQ-2 $[15,16]$ were similar at around $10 \%$ but MSM were more likely than MSEW to report 
Table 2 Sexual partner numbers in the lifetime, past 5 years, and past year reported by men who have sex exclusively with women (MSEW), all men who have sex with men (MSM), and MSM who identified as gay in Natsal-3

\begin{tabular}{|c|c|c|c|c|c|c|c|c|c|}
\hline \multirow[b]{3}{*}{ Denominators ${ }^{a}$ : } & \multicolumn{3}{|c|}{ All sexual partners (regardless of gender), reported by: } & \multicolumn{3}{|c|}{ Male sexual partners, reported by: } & \multicolumn{3}{|c|}{ Female sexual partners, reported by: } \\
\hline & MSEW & All MSM & $\begin{array}{l}\text { MSM who identified } \\
\text { as gay }\end{array}$ & MSEW & All MSM & $\begin{array}{l}\text { MSM who identified } \\
\text { as gay }{ }^{b}\end{array}$ & MSEW & All MSM & $\begin{array}{l}\text { MSM who identified } \\
\text { as gay }{ }^{b}\end{array}$ \\
\hline & 5069,6257 & 190,191 & 107,99 & 5069,6257 & 190,191 & 107,99 & 5069,6257 & 190,191 & 107,99 \\
\hline \multicolumn{10}{|l|}{ Timeframe: } \\
\hline \multicolumn{10}{|l|}{ Lifetime } \\
\hline \multicolumn{10}{|l|}{ Age standardised: } \\
\hline \multicolumn{10}{|l|}{$\%[95 \% \mathrm{Cl}]$} \\
\hline 0 & 0 & 0 & 0 & $97.5[97.0-98.0]$ & 0 & 0 & 0 & $30.1[23-38.3]$ & $55.4[42.7-67.4]$ \\
\hline 1 & $13.7[12.6-14.9]$ & $1.8[0.7-4.5]$ & $1.3[0.3-5.3]$ & $1.4[1.1-1.9]$ & $14.6[9.3-22.2]$ & $3.1[0.8-10.7]$ & $13.7[12.6-14.9]$ & $13.7[9.0-20.3]$ & $22.1[13.6-33.8]$ \\
\hline 2 & $8.4[7.5-9.3]$ & $1.8[0.9-3.8]$ & $1.5[0.5-4.9]$ & $0.5[0.4-0.8]$ & $9.3[5.2-16.0]$ & $4.4[1.8-10.4]$ & $8.4[7.5-9.4]$ & $8.5[4.6-15.0]$ & $8.8[3.5-20.4]$ \\
\hline $3-4$ & $15.3[14.1-16.5]$ & $9.2[5.2-15.9]$ & 8 [3.8-16.3] & $0.3[0.1-0.5]$ & 15.1 [9.8-22.3] & $10[5.1-18.8]$ & 15.6 [14.4-16.8] & $9.6[5.7-15.9]$ & $6.2[2.3-15.3]$ \\
\hline $5-9$ & $25.7[24.3-27.1]$ & 19.1 [13.3-26.6] & $18.1[11.3-27.5]$ & $0.2[0.1-0.4]$ & $18.5[12.3-27]$ & $12.9[7.6-21.0]$ & $25.4[24-26.8]$ & $11.3[6.6-18.7]$ & $4.8[1.8-11.9]$ \\
\hline $10+$ & 37 [35.5-38.6] & 68 [59.5-75.4] & 71.1 [61.3-79.3] & $0.1[0-0.3]$ & $42.5[34.3-51.2]$ & 69.6 [59.9-77.9] & $37[35.5-38.5]$ & $26.9[19.5-35.8]$ & $2.8[1.1-6.8]$ \\
\hline Mean & 14.3 & 111.1 & 86.7 & 0.1 & 45.7 & 84.8 & 14.3 & 60.4 & 1.2 \\
\hline $\mathrm{aAOR}^{\mathrm{c}}$ & 1.00 & $3.40(2.42-4.78)$ & $3.42(2.25-5.22)$ & 1.00 & $836(516-1356)$ & $2504(1191-5267)$ & 1.00 & $0.15(0.07-0.32)$ & $0.00(0.00-0.01)$ \\
\hline \multicolumn{10}{|c|}{ Non-age standardised: } \\
\hline Mean (sd) & $14.3(43.1)$ & $90.1(345.0)$ & $76.4(166.6)$ & $0.1(0.5)$ & $44.7(121.6)$ & $74.2(165.5)$ & $14.3(43.1)$ & $44.6(323.6)$ & $1.3(3.2)$ \\
\hline Median (IQR) & $6(3-15)$ & $17(6-50)$ & $19(6-70)$ & $0(0-0)$ & $6(2-25)$ & $19(5-70)$ & $6(3-5)$ & $2(0-9)$ & $0(0-1)$ \\
\hline \multicolumn{10}{|l|}{ Past 5 years } \\
\hline \multicolumn{10}{|c|}{ Age standardised: } \\
\hline \multicolumn{10}{|l|}{$\%[95 \% \mathrm{Cl}]$} \\
\hline 0 & 0 & 0 & 0 & N/A & & & 0 & $54.1[45.7-62.3]$ & 93.5 [89.6-96.1] \\
\hline 1 & $62.7[61.3-64.0]$ & 15.6 [10.1-23.3] & $26.7[16.5-40.3]$ & & $32.2[24.2-41.5]$ & 26.3 [16.2-39.8] & $62.7[61.3-64]$ & 16.1 [10.5-23.9] & $3.3[1.6-6.5]$ \\
\hline 2 & $11.4[10.5-12.4]$ & $10.7[6.4-17.5]$ & $5.5[2.5-11.9]$ & & $13.5[8.5-20.8]$ & 6.8 [3.4-13.0] & $11.4[10.5-12.4]$ & $5.5[2.5-11.3]$ & $1.4[0.4-4.7]$ \\
\hline $3-4$ & 11.3 [10.4-12.2] & 15.3 [10-22.8] & $19.4[11-31.7]$ & & $15.7[10.4-23.1]$ & 19.1 [10.9-31.3] & 11.3 [10.4-12.2] & $9.7[5.3-16.9]$ & $1.4[0.4-4.7]$ \\
\hline $5-9$ & $8.4[7.7-9.2]$ & $22.6[16.4-30.3]$ & $13.5[7.1-24.3]$ & & $11.9[7.5-18.3]$ & $12.6[6.4-23.3]$ & $8.4[7.7-9.2]$ & $6.1[3.1-11.7]$ & $0.4[0-2.5]$ \\
\hline $10+$ & $6.3[5.7-6.9]$ & 35.8 [27.8-44.8] & 34.8 [23.7-47.9] & & 26.7 [19.7-35.2] & $35.3[24.2-48.1]$ & $6.3[5.7-6.9]$ & $8.5[4.7-15.2]$ & 0 \\
\hline Mean & 3 & 15.8 & 17.3 & & 12.2 & 17.5 & 3.0 & 3.7 & 0.1 \\
\hline $\mathrm{aAOR}^{\mathrm{c}}$ & 1.00 & $9.36(6.23-14.08)$ & $6.22(3.57-10.84)$ & & & & 1.00 & $0.01(0.00-0.03)$ & $0.00(0.00-0.00)$ \\
\hline
\end{tabular}


Table 2 Sexual partner numbers in the lifetime, past 5 years, and past year reported by men who have sex exclusively with women (MSEW), all men who have sex with men (MSM), and MSM who identified as gay in Natsal-3 (Continued)

\begin{tabular}{|c|c|c|c|c|c|c|c|c|c|}
\hline \multicolumn{10}{|c|}{ Non-age standardised: } \\
\hline Mean (sd) & $3.0(5.3)$ & $15.9(35.2)$ & $18.1(43.5)$ & & $12.3(31.7)$ & $18.3(43.7)$ & $3.0(5.3)$ & $3.6(16.6)$ & $0.2(0.7)$ \\
\hline Median (IQR) & $1(1-3)$ & $6(2-16)$ & $5(2-30)$ & & $3(1-10)$ & $5(2-30)$ & $1(1-3)$ & $0(0-2)$ & $0(0-0)$ \\
\hline \multicolumn{4}{|l|}{ Past year } & $\mathrm{N} / \mathrm{A}$ & & & & & \\
\hline \multicolumn{10}{|c|}{ Age standardised: } \\
\hline \multicolumn{10}{|l|}{$\%[95 \% \mathrm{Cl}]$} \\
\hline 0 & $6.1[5.4-6.9]$ & $3.9[1.9-8.1]$ & $6[2.2-15.5]$ & & $14.9[9.6-22.2]$ & $5.9[2.2-15.3]$ & $6.1[5.4-6.9]$ & $60.8[51.8-69.1]$ & 97.7 [94.1-99.2] \\
\hline 1 & 77.1 [75.8-78.4] & $36.0[27.8-45.1]$ & 45.8 [33.2-59.0] & & $42.6[33.9-51.7]$ & $45.3[32.8-58.4]$ & 77.1 [75.8-78.4] & $21.6[15.0-30.2]$ & $0.8[0.1-5.1]$ \\
\hline 2 & $8.0[7.2-8.8]$ & $13[8.0-20.4]$ & $8.5[3.9-17.6]$ & & $9.7[5.8-15.7]$ & $9.0[4.3-17.9]$ & $8.0[7.2-8.8]$ & $6.2[3.2-11.8]$ & $0.7[0.2-2.9]$ \\
\hline $3-4$ & $5.4[4.8-6.1]$ & 16.1 [10.8-23.5] & $12.8[7-22.4]$ & & 13.1 [8.6-19.6] & 13.9 [7.7-23.8] & $5.4[4.8-6.1]$ & $7.1[3.7-13.0]$ & $0.7[0.1-5.1]$ \\
\hline $5-9$ & $2.2[1.9-2.7]$ & $17.6[11.7-25.5]$ & $7.3[2.8-17.4]$ & & $8.6[4.7-15.3]$ & $6.4[2.2-16.9]$ & $2.2[1.9-2.7]$ & $3.7[1.5-9.0]$ & 0 \\
\hline $10+$ & $1.2[0.9-1.5]$ & $13.4[8.0-21.7]$ & 19.6 [10.4-34.0] & & $11.2[6.4-18.8]$ & 19.5 [10.4-33.7] & $1.2[0.9-1.5]$ & $0.7[0.1-4.5]$ & 0 \\
\hline Mean & 1.4 & 4.5 & 5.0 & & 3.5 & 4.9 & 1.4 & 0.9 & 0 \\
\hline $\mathrm{aAOR}^{\mathrm{c}}$ & 1.00 & $8.29(5.25-13.09)$ & $4.84(2.50-9.37)$ & & & & 1.00 & $0.04(0.02-0.08)$ & $0.00(0.00-0.00)$ \\
\hline \multicolumn{10}{|c|}{ Non-age standardised: } \\
\hline Mean (sd) & $1.4(2.0)$ & $4.5(8.5)$ & $5.1(11.7)$ & & $3.5(8.4)$ & $4.9(11.7)$ & $1.5(2.0)$ & $0.9(1.8)$ & $0.1(0.4)$ \\
\hline Median (IQR) & $1(1-1)$ & $2(1-5)$ & $1(1-4)$ & & $1(1-4)$ & $1(1-4)$ & $1(1-1)$ & $0(0-1)$ & $0(0-0)$ \\
\hline New partner, past & & & & N/A & & & & & \\
\hline \multicolumn{10}{|c|}{ Age standardised: } \\
\hline \multicolumn{10}{|l|}{$\%[95 \%$ Cl] } \\
\hline 0 & 77.7 [76.6-78.8] & $43.3[34.8-52.2]$ & $49.8[37.1-62.5]$ & & $55.7[47.0-64.1]$ & $50.8[38.1-63.4]$ & 77.7 [76.6-78.8] & $80.1[72.4-86.1]$ & 97.7 [94.1-99.2] \\
\hline 1 & $13.2[12.3-14.2]$ & 14.8 [10.1-21.3] & $15.6[9.3-25.1]$ & & 13.1 [9.1-18.6] & $15.6[9.3-25.0]$ & 13.2 [12.3-14.2] & $9.6[5.6-16.0]$ & $1.9[0.6-5.7]$ \\
\hline 2 & $4.5[4.0-5.1]$ & $7.8[4.1-14.2]$ & $4.5[1.7-11.6]$ & & $6.1[3.3-11.0]$ & $4.8[1.9-11.8]$ & $4.5[4.0-5.1]$ & 5.2 [2.7-9.9] & $0.4[0.0-2.5]$ \\
\hline $3-4$ & $2.7[2.3-3.1]$ & 12.3 [7.9-18.8] & $9.5[4.3-19.5]$ & & $9.7[6.0-15.4]$ & $9.3[4.1-19.8]$ & $2.7[2.3-3.1]$ & $2.7[0.9-8.1]$ & 0 \\
\hline $5-9$ & $1.4[1.1-1.7]$ & $15.6[9.5-24.5]$ & $14.1[6.1-29.2]$ & & $10.0[5.0-18.8]$ & $13.1[5.4-28.4]$ & $1.4[1.1-1.7]$ & $1.7[0.5-6.0]$ & 0 \\
\hline $10+$ & $0.5[0.4-0.7]$ & $6.2[3.2-11.5]$ & $6.5[2.9-13.9]$ & & 5.4 [2.7-10.5] & 6.4 [2.9-13.6] & $0.5[0.4-0.7]$ & $0.7[0.1-4.5]$ & 0 \\
\hline Mean & 0.5 & 3.2 & 3.6 & & 2.6 & 3.5 & 0.5 & 0.5 & 0 \\
\hline $\mathrm{aAOR}^{\mathrm{c}}$ & 1.00 & $6.24(3.86-10.08)$ & $4.32(2.35-7.95)$ & & & & 1.00 & $0.76(0.47-1.23)$ & $0.08(0.03-0.22)$ \\
\hline
\end{tabular}


Table 2 Sexual partner numbers in the lifetime, past 5 years, and past year reported by men who have sex exclusively with women (MSEW), all men who have sex with men (MSM), and MSM who identified as gay in Natsal-3 (Continued)

\begin{tabular}{|c|c|c|c|c|c|c|c|c|c|}
\hline \multicolumn{10}{|c|}{ Non-age standardised: } \\
\hline Mean (sd) & $0.5(1.4)$ & $3.2(8.5)$ & $3.9(11.8)$ & & $2.6(8.3)$ & $3.7(11.8)$ & $0.5(1.4)$ & $0.5(1.6)$ & $0.0(0.2)$ \\
\hline Median (IQR) & $0(0-0)$ & $1(0-4)$ & $1(0-3)$ & & & $1(0-3)$ & $0(0-0)$ & $0(0-0)$ & $0(0-0)$ \\
\hline \multicolumn{4}{|c|}{ Number of condomless-sex partners, past year } & N/A & & & & & \\
\hline \multicolumn{10}{|l|}{ Age standardised: } \\
\hline \multicolumn{10}{|l|}{$\%[95 \% \mathrm{Cl}]$} \\
\hline 0 & $19.5[18.3-20.9]$ & $43.1[34.4-52.3]$ & $43.4[32.3-55.3]$ & & $42.9[32.6-54.0]$ & $30.4[20.0-43.3]$ & $19.3[18.1-20.6]$ & $73.7[65.5-80.5]$ & 98.8 [95.0-99.7] \\
\hline 1 & $72.4[70.9-73.8]$ & $32.2[24.5-41.0]$ & $40.1[29.2-52.1]$ & & $37.1[27.4-48.1]$ & $49.7[36.6-62.9]$ & $72.7[71.3-74.1]$ & $14.7[9.5-22.1]$ & $1.2[0.3-5.0]$ \\
\hline $2+$ & $8.0[7.3-8.9]$ & $24.7[17.6-33.4]$ & $16.4[8.8-28.8]$ & & $19.9[11.7-31.9]$ & $19.8[10.4-34.6]$ & $7.9[7.2-8.8]$ & $11.6[7.3-17.9]$ & 0 \\
\hline Mean & 1.0 & 1.2 & 1.0 & & 1.1 & 1.3 & 1.0 & 0.5 & 0.01 \\
\hline $\mathrm{aAOR}^{\mathrm{c}}$ & 1.00 & $0.60(0.30-1.18)$ & $0.43(0.21-0.88)$ & & & & 1.00 & $0.10(0.06-0.16)$ & $0.00(0.00-0.01)$ \\
\hline \multicolumn{10}{|c|}{ Non-age standardised: } \\
\hline Mean $(s d)$ & $1.0(1.0)$ & $1.2(2.6)$ & $1.2(3.1)$ & & $1.2(2.7)$ & $1.5(3.2)$ & $1.0(1.0)$ & $0.5(1.4)$ & $0.01(0.1)$ \\
\hline Median (IQR) & $1(1-1)$ & $1(0-1)$ & $1(0-1)$ & & $1(0-1)$ & $1(0-1)$ & $1(1-1)$ & $0(0-1)$ & $0(0-0)$ \\
\hline
\end{tabular}

anweighted, weighted denominators defined as participants who reported at least one sexual partner in the 5 years prior to interview

${ }^{\mathrm{b}} \mathrm{MSM}$ who identified as gay in Natsal-3 are a subset of all MSM

'Age-adjusted odds ratio (aAOR) for comparing to MSEW 
Table 3 Sociodemographic profile of men who have sex exclusively with women (MSEW), all men who have sex with men (MSM), and MSM who identified as gay in Natsal-3

\begin{tabular}{|c|c|c|c|c|c|}
\hline \multirow{3}{*}{ Denominators $^{a}$ : } & MSEW & All MSM & & MSM who identified as gay ${ }^{b}$ & \multirow[b]{3}{*}{$p$-value } \\
\hline & 5069,6257 & 190,191 & \multirow[b]{2}{*}{$p$-value* } & \multirow{2}{*}{107,99} & \\
\hline & $\%[95 \% \mathrm{Cl}]$ & $\%[95 \% \mathrm{Cl}]$ & & & \\
\hline \multicolumn{6}{|l|}{ Age group } \\
\hline $16-34$ & $35.0[33.6-36.5]$ & $43.6[35.9-51.6]$ & \multirow[t]{4}{*}{0.106} & $51.3[40.2-62.3]$ & \multirow[t]{4}{*}{$<0.001$} \\
\hline $35-54$ & $41.0[39.3-42.8]$ & $38.3[30.3-47.0]$ & & $41.2[30.2-53.1]$ & \\
\hline $55-74$ & $23.9[22.5-25.4]$ & $18.1[12.1-26.2]$ & & $7.5[3.7-14.8]$ & \\
\hline \multirow[t]{2}{*}{ Median (IQR) } & $41(30-54)$ & $40(26-51)$ & & $34(26-46)$ & \\
\hline & \multicolumn{3}{|c|}{ Age-standardised estimates: } & & \\
\hline \multicolumn{6}{|l|}{ Relationship status } \\
\hline Married/civil partnership & $54.4[53.1-55.7]$ & $25.6[18.2-34.8]$ & \multirow[t]{4}{*}{$<0.001$} & $14.9[8.0-26.0]$ & \multirow[t]{4}{*}{$<0.001$} \\
\hline Living with partner & $16.0[14.9-17.1]$ & $20.6[14.3-28.8]$ & & $21.8[12.8-34.7]$ & \\
\hline In a 'steady' relationship but not living together & $11.8[11.0-12.7]$ & $14.5[9.9-20.7]$ & & $15.8[9.5-25.0]$ & \\
\hline Not in a 'steady' relationship & $17.8[16.9-18.8]$ & $39.3[31.5-47.6]$ & & $47.6[35.5-59.9]$ & \\
\hline Ethnicity - white & $88.4[87.2-89.5]$ & $96.8[91.6-98.8]$ & 0.007 & 98.9 [92.9-99.8] & 0.013 \\
\hline Religion/religious beliefs -fairly/very important & $33.8[32.3-35.4]$ & $23.4[16.9-31.4]$ & 0.007 & $27.3[17.6-39.9]$ & 0.056 \\
\hline \multicolumn{6}{|l|}{ Academic qualifications $^{c}$} \\
\hline No academic qualifications & $17.9[16.7-19.2]$ & $10.2[6.0-16.9]$ & \multirow[t]{3}{*}{0.017} & $9.3[4.2-19.5]$ & \multirow[t]{3}{*}{0.010} \\
\hline Academic qualifications typically gained at age 16 & $34.3[32.8-35.8]$ & $33.8[26.2-42.2]$ & & $31.7[21.2-44.5]$ & \\
\hline Studying for/attained further academic qualifications & $47.8[46.1-49.5]$ & $56.0[47-64.6]$ & & $58.9[45.7-71.0]$ & \\
\hline \multicolumn{6}{|l|}{ National Statistics Socio-Economic Classification ${ }^{d}$} \\
\hline Managerial \& professional occupations & $38.1[36.4-39.7]$ & $43.1[34.6-52]$ & \multirow[t]{5}{*}{0.086} & $43.8[31.9-56.5]$ & \multirow[t]{5}{*}{0.094} \\
\hline Intermediate occupations & $17.1[15.8-18.4]$ & 20.0 [13.7-28.3] & & $26.5[16.4-39.8]$ & \\
\hline Semi-routine/routine occupations & $32.6[31.1-34.2]$ & $27.5[20.1-36.4]$ & & 18.9 [11.9-28.8] & \\
\hline Never worked and long-term unemployed & $5.0[4.4-5.7]$ & $1.2[0.4-3.9]$ & & $3.7[0.9-13.7]$ & \\
\hline Full-time students & $7.2[6.5-8]$ & $8.2[54-12.3]$ & & $7.1[3.8-12.8]$ & \\
\hline Lives in an urban area & $76.6[74.8-78.3]$ & $81.5[72.9-87.9]$ & 0.118 & $89.1[79.9-94.4]$ & 0.005 \\
\hline \multicolumn{6}{|l|}{ Quintile of Index of Multiple Deprivation ${ }^{f}$} \\
\hline 1 (least deprived) & $21.2[19.7-22.8]$ & $18.9[12.7-27.2]$ & \multirow[t]{5}{*}{0.530} & $13.1[7.5-22.0]$ & \multirow[t]{5}{*}{0.116} \\
\hline 2 & $21.6[20.1-23.2]$ & $16.6[11.0-24.3]$ & & $17.2[9.2-29.8]$ & \\
\hline 3 & $19.4[17.9-20.9]$ & $21.0[15.0-28.7]$ & & $16.9[10.1-26.8]$ & \\
\hline 4 & $19.6[18.1-21.2]$ & $21.2[15.1-29.0]$ & & $26.2[17.3-37.6]$ & \\
\hline 5 (most deprived) & $18.2[16.8-19.6]$ & $22.2[16.2-29.6]$ & & $26.6[18.6-36.6]$ & \\
\hline
\end{tabular}

${ }^{*} p$-value for comparing all MSM to MSEW

${ }^{* *} p$-value for comparing MSM who identified as gay in Natsal-3 to MSEW. Note that MSM who identify as gay are a subset of all MSM

a Unweighted, weighted denominators defined as participants who reported at least one sexual partner in the 5 years prior to interview

${ }^{\mathrm{b}} \mathrm{MSM}$ who identified as gay in Natsal-3 are a subset of all MSM

'Participants aged $\geq 17$ years

${ }^{\mathrm{d}}$ National Statistics Socio-Economic Classification is a measure of individual socio-economic status [17]

e Defined as areas with a population of at least 10,000 people

fIndex of Multiple Deprivation is an area-level measure of deprivation [18]

having received treatment for depression (past year, aAOR: 2.75).

\section{Sexual behaviours and sexual health outcomes}

In addition to MSM being more likely than MSEW to report larger numbers of partners (Table 2), including reporting ten or more partners (aAOR:11.69, Table 5) and condomless sex with two or more partners (aAOR: 3.52 ), both in the past year, they were also more likely to have had concurrent partners in the past five years (aAOR:5.65). However, there was no difference in the proportions reporting condomless first sex with their 
Table 4 Health behaviours and outcomes reported by men who have sex exclusively with women (MSEW), all men who have sex with men (MSM) and MSM who identified as gay in Natsal-3

\begin{tabular}{|c|c|c|c|c|c|}
\hline & MSEW & All MSM & & MSM who identified as gay ${ }^{b}$ & \\
\hline \multirow[t]{2}{*}{ Denominators: ${ }^{a}$} & 5069,6257 & 190, 191 & & 107,99 & \\
\hline & $\%[95 \% \mathrm{Cl}]$ & $\%[95 \% \mathrm{Cl}]$ & $p$-value ${ }^{*}$ & $\%[95 \% \mathrm{Cl}]$ & $p$-value \\
\hline \multicolumn{6}{|l|}{ Health behaviours } \\
\hline Usual alcohol consumption more than recommended levels ${ }^{c}$ & $8.8[8-9.8]$ & $11.4[7.2-17.6]$ & & $10.3[5.7-18.0]$ & \\
\hline aAOR $[95 \% \mathrm{Cl}]^{\mathrm{e}}$ & 1.00 & $1.43[0.87-2.36]$ & 0.156 & $1.67[0.87-3.21]$ & 0.125 \\
\hline \multicolumn{6}{|l|}{ Usual frequency of binge drinking ${ }^{d}$} \\
\hline Never or rarely & $60.7[59.1-62.3]$ & $54.0[44.9-62.9]$ & & $58.6[48-68.5]$ & \\
\hline Monthly & $17.5[16.4-18.8]$ & $24.9[17.3-34.4]$ & & $25.4[16.6-36.8]$ & \\
\hline Weekly or daily & $21.7[20.4-23.1]$ & $21.1[15.1-28.7]$ & & $16.0[9.9-24.8]$ & \\
\hline aAOR $[95 \% \mathrm{Cl}]^{\mathrm{e}, \mathrm{f}}$ & 1.00 & $0.99[0.66-1.49]$ & 0.972 & $0.84[0.49-1.45]$ & 0.535 \\
\hline \multicolumn{6}{|l|}{ Smoking status } \\
\hline Never & $47.2[45.6-48.9]$ & $29.5[22.7-37.4]$ & & $30.2[21.5-40.6]$ & \\
\hline Ex-smoker & $25.9[24.5-27.4]$ & $32.6[25.2-41]$ & & 38.9 [28.8-49.9] & \\
\hline Current smoker & $26.8[25.4-28.3]$ & $37.9[30-46.4]$ & & $30.9[22.1-41.4]$ & \\
\hline aAOR $\left[95 \%\right.$ Cl] ${ }^{e, f}$ & 1.00 & $1.72[1.21-2.45]$ & 0.003 & $1.37[0.87-2.16]$ & 0.173 \\
\hline \multicolumn{6}{|l|}{ Recreational drug use, past year } \\
\hline None & $84.3[83.2-85.3]$ & $64.0[56-71.3]$ & & $59.1[47.4-69.9]$ & \\
\hline Cannabis only & $9.1[8.3-9.9]$ & $10.2[6.1-16.7]$ & & $8.1[3.2-19]$ & \\
\hline Cannabis and/or other drugs & $6.7[6.0-7.4]$ & $25.8[19.3-33.6]$ & & $32.8[23.9-43.2]$ & \\
\hline aAOR $[95 \% \mathrm{Cl}]^{\mathrm{e}, \mathrm{f}}$ & 1.00 & $3.46[2.22-5.40]$ & $<0.001$ & $4.18[2.33-7.49]$ & $<0.001$ \\
\hline Injecting drug use, ever & $1.3[1.0-1.7]$ & $0.7[0.2-2.8]$ & & $0.8[0.1-5.4]$ & \\
\hline aAOR $[95 \% \mathrm{Cl}]^{\mathrm{e}}$ & 1.00 & $0.49[0.11-2.08]$ & 0.332 & $0.53[0.07-3.90]$ & 0.529 \\
\hline $\begin{array}{l}\text { Taken any medications that have limited your sexual activity } \\
\text { or enjoyment, past year }\end{array}$ & $7.8[7.0-8.7]$ & 16.8 [11.3-24.2] & & $21.5[12.8-33.9]$ & \\
\hline aAOR $[95 \% \mathrm{Cl}]^{\mathrm{e}}$ & 1.00 & $2.56[1.57-4.19]$ & $<0.001$ & $3.24[1.77-5.94]$ & $<0.001$ \\
\hline \multicolumn{6}{|l|}{ Health outcomes } \\
\hline Self-reported health status ['bad'/'very bad'] & $3.1[2.7-3.7]$ & $5.2[2.7-9.7]$ & & $9.5[4.1-20.5]$ & \\
\hline aAOR $[95 \% \mathrm{Cl}]^{\mathrm{e}}$ & 1.00 & $1.67[0.88-3.18]$ & 0.117 & $2.23[0.99-5.01]$ & 0.052 \\
\hline$\geq 1$ long-standing illness/disability/infirmity & $29.9[28.5-31.4]$ & $38.1[30.8-45.9]$ & & $44.1[34.7-53.9]$ & \\
\hline aAOR $[95 \% \mathrm{Cl}]^{\mathrm{e}}$ & 1.00 & $1.46[1.02-2.09]$ & 0.041 & $1.65[1.03-2.67]$ & 0.039 \\
\hline \multicolumn{6}{|l|}{ Number of chronic conditionsh } \\
\hline 0 & $68.7[67.2-70.1]$ & $66[57.5-73.6]$ & & $58.4[50.4-65.9]$ & \\
\hline 1 & $20.6[19.3-22.0]$ & $20.2[14.0-28.2]$ & & $22.7[13.6-35.4]$ & \\
\hline$\geq 2$ & $10.7[9.8-11.8]$ & $13.8[8.8-21.0]$ & & $18.9[11.0-30.7]$ & \\
\hline aAOR $[95 \% \mathrm{Cl}]^{\mathrm{e}, \mathrm{f}}$ & 1.00 & $1.16[0.75-1.81]$ & 0.67 & $1.31[0.79-2.18]$ & 0.293 \\
\hline \multicolumn{6}{|l|}{ Body Mass Indexi } \\
\hline Healthy: $18.5-24.9 \mathrm{~kg} / \mathrm{m}^{2}$ & $40.6[39.1-42.1]$ & $46.1[37.2-55.2]$ & & $47.5[35.8-59.5]$ & \\
\hline Underweight: $<18.5 \mathrm{~kg} / \mathrm{m}^{2}$ & $1.1[0.9-1.5]$ & $2.7[1.3-5.3]$ & & $2.4[0.9-6.1]$ & \\
\hline Overweight: $25-30 \mathrm{~kg} / \mathrm{m}^{2}$ & $40.0[38.4-41.6]$ & $28.4[21.1-36.9]$ & & $37.3[26.9-49.0]$ & \\
\hline Obese: $>30 \mathrm{~kg} / \mathrm{m}^{2}$ & $18.3[16.5-20.3]$ & $22.9[13.5-38.2]$ & & $12.8[4.6-29.6]$ & \\
\hline aAOR $[95 \% \mathrm{Cl}]^{\mathrm{e}, \mathrm{f}}$ & 1.00 & $0.77[0.54-1.11]$ & 0.156 & $0.68[0.44-1.07]$ & 0.094 \\
\hline Screened positive for depression at interview & $8.9[8.1-9.8]$ & $8.9[5.5-14.3]$ & & $6.4[3.5-11.7]$ & \\
\hline aAOR $[95 \% \mathrm{Cl}]^{\mathrm{e}}$ & 1.00 & $1.03[0.62-1.70]$ & 0.909 & $0.92[0.48-1.77]$ & 0.797 \\
\hline
\end{tabular}


Table 4 Health behaviours and outcomes reported by men who have sex exclusively with women (MSEW), all men who have sex with men (MSM) and MSM who identified as gay in Natsal-3 (Continued)

\begin{tabular}{lllll}
\hline $\begin{array}{l}\text { Received treatment for depression from a health professional, } \\
\text { past year }\end{array}$ & $5.8[5.1-6.5]$ & $14.0[9.2-20.5]$ & $19.9[11.9-31.4]$ \\
$\quad$ aAOR $[95 \% \mathrm{Cl}]^{\mathrm{e}}$ & 1.00 & $2.75[1.69-4.47]$ & $<0.001$ & $4.03[2.30-7.07]$ \\
$\begin{array}{l}\text { Any health condition/disability that has affected sexual } \\
\text { activity/enjoyment, past year }\end{array}$ & $15.3[14.2-16.5]$ & $27.1[19.9-35.7]$ & $29.3[19.2-42.1]$ \\
$\quad$ aAOR [95\% Cl]
\end{tabular}

All estimates are age-standardised

${ }^{*} p$-value for comparing all MSM to MSEW

${ }^{* *} p$-value for comparing MSM who identified as gay in Natsal-3 to MSEW. Note that MSM who identify as gay are a subset of all MSM

anweighted, weighted denominators defined as participants who reported at least one sexual partner in the 5 years prior to interview

${ }^{b}$ MSM who identified as gay in Natsal-3 are a subset of all MSM

'Defined as usual alcohol consumption greater than recommended levels of 21 units per week [34]

${ }^{\mathrm{d}}$ Defined as usually drinking more than 8 units of alcohol on one occasion [34]

$\mathrm{e}_{\mathrm{aAOR}}=$ age-adjusted odds ratio

${ }^{f}$ Age-adjusted odds ratio for reporting the responses in bold font [for those variables with $>2$ response options] relative to MSEW

IIn contrast to reporting 'very good', 'good', or 'fair'

${ }^{\mathrm{h}}$ Measure of comorbidity includes arthritis, heart attack, coronary heart disease, angina, other forms of heart disease, hypertension, stroke, diabetes, broken hip or pelvis bone or hip replacement ever, backache lasting $>3$ months, any other muscle or bone disease lasting $>3$ months, cancer, and any thyroid condition treated in the past year [4]

'Body Mass Index thresholds as per National Obesity Observatory/Public Health England [35]

${ }^{\mathrm{j}}$ As per $\mathrm{PHQ}-2[15,16]$

three most recent partners (past year). Almost one in five MSM reported taking drugs to assist sexual performance in contrast to $6.5 \%$ of MSEW (past year, aAOR:3.50).

MSM perceived greater STI/HIV risk than MSEW, although only a minority of MSM considered themselves 'quite a lot'/'greatly' at risk of either STIs or HIV. Similarly, a minority of MSM reported sexual health clinic attendance (33.0\%), testing for HIV (17.0\%), and STI diagnosis/es (4.9\%), each in the past year, but these were all more commonly reported by MSM than MSEW, including after adjusting for key sociodemographics, healthrelated factors, and partner numbers (AORs: 4.71, 6.08, and 5.67, respectively).

While MSM were as likely as MSEW to report feeling distressed/worried about their sex lives, MSM were more likely than MSEW to report seeking professional help/advice for their sex life in the past year, and specifically from sexual health/GUM/STI clinics if they did (data not shown). Among sexually-active men, MSM were more likely than MSEW to have poorer overall sexual function according to the Natsal-SF [14] (AOR:2.28).

Among all MSM, $22.4 \%$ reported having ever experienced attempted non-volitional sex, with the corresponding proportion estimate significantly smaller for MSEW at $4.3 \%$ differences that remained in multivariable analyses (AOR:6.51).

\section{Clustering of harmful health behaviours and poor health outcomes}

MSM were not only more likely than MSEW to report a number of harmful behaviours, but also to do so in combination within both the physical and sexual health domains (Table 6; corresponding aAORs:2.09 [95 \% CI: 1.55-2.82] and 3.40 [95 \% CI:2.10-5.48], respectively).
Furthermore, proportional Venn diagrams show that MSM were more likely than MSEW to report harmful behaviours across the physical health and sexual health domains, with $25.9 \%$ of MSM in comparison to $10.4 \%$ of MSEW reporting harmful behaviours in both domains.

There was no difference in the number of poor physical health outcomes reported by MSM and MSEW (aAOR:1.02, 95 \% CI:0.71-1.48, Table 7). In contrast MSM were more likely than MSEW to report one or more of the poor sexual health outcomes considered and to do so in combination within this domain (aAOR: 3.42, 95 \% CI:2.30-5.08). Furthermore, a larger proportion of MSM reported poor health outcomes across multiple domains $(32.7 \%, 95 \%$ CI:24.8 \%-41.7 \%) relative to MSEW (15.0 \%, $95 \%$ CI:13.9 \%-16.2 \%), with $8.4 \%$ of all MSM having experienced poor health outcomes in all three domains - physical, mental, and sexual health in contrast to $1.5 \%$ of all MSEW.

\section{Summary comparing gay-identifying MSM to MSEW}

When comparing gay-identifying MSM to MSEW, we observed similar patterns to those described above when comparing all MSM to MSEW, reflecting how gayidentifying MSM constitute half the sample of all MSM. Some differences exist though. Gay-identifying MSM were younger than MSEW, with half aged under 35, and so were also less likely to be in a steady or cohabiting relationship. Gay-identifying MSM were more likely than MSEW to live in urban areas, and often areas of greatest deprivation. The health behaviours of gay-identifying MSM differed to MSEW in similar ways to those observed for all MSM relative to MSEW, especially regarding greater reporting of recreational drug use. However, the prevalence of the adverse health outcomes studied 
Table 5 Sexual behaviours, risk perception, and sexual health outcomes reported by men who have sex exclusively with women (MSEW), all men who have sex with men (MSM), and MSM who identified as gay in Natsal-3

\begin{tabular}{|c|c|c|c|c|c|}
\hline \multirow{3}{*}{ Denominators: ${ }^{a}$} & MSEW & All MSM & & MSM who identified as gay & \multirow[b]{3}{*}{$p$-value } \\
\hline & 5069,6257 & 190,191 & \multirow[b]{2}{*}{$p$-value } & $\begin{array}{l}107,99 \\
\%[95 \%\end{array}$ & \\
\hline & $\%[95 \% \mathrm{Cl}]$ & $\%[95 \% \mathrm{Cl}]$ & & $\%[95 \% \mathrm{Cl}]$ & \\
\hline \multicolumn{6}{|l|}{ Sexual behaviours } \\
\hline$\geq 10$ partners $^{\mathrm{c}}$, past year & $1.2[0.9-1.5]$ & $13.4[8.0-21.6]$ & & $19.6[10.5-33.7]$ & \\
\hline aAOR $[95 \% \mathrm{Cl}]^{\mathrm{d}}$ & 1.00 & $11.69[6.06-22.54]$ & $<0.001$ & $15.91[7.10-35.66]$ & $<0.001$ \\
\hline $\begin{array}{l}\text { Non-use of condoms at first sex with } \\
\text { any of the three most recent partners } \\
\text { past year }\end{array}$ & $10.1[9.3-11]$ & $15.2[9.7-23.1]$ & & $6.0[3.2-10.9]$ & \\
\hline aAOR $[95 \% \mathrm{Cl}]^{d}$ & 1.00 & $1.50[0.91-2.49]$ & 0.114 & $0.66[0.35-1.24]$ & 0.198 \\
\hline Condomless sex with $\geq 2$ partners ${ }^{c}$, past year & $8.1[7.3-8.9]$ & $24.6[17.1-34.1]$ & & $13.0[6.6-24.3]$ & \\
\hline aAOR $[95 \% \mathrm{Cl}]^{\mathrm{d}}$ & 1.00 & $3.52[2.13-5.83]$ & $<0.001$ & $1.86[0.85-4.07]$ & 0.120 \\
\hline Concurrent partners ${ }^{c}$, past 5 years & $15.6[14.5-16.7]$ & $52.4[43.5-61.1]$ & & $41.6[29.7-54.5]$ & \\
\hline aAOR $[95 \% \mathrm{Cl}]^{\mathrm{d}}$ & 1.00 & $5.65[3.85-8.30]$ & $<0.001$ & $3.66[2.23-6.00]$ & $<0.001$ \\
\hline Taken drugs to assist sexual performance, past year & $6.5[5.7-7.3]$ & $19.1[12.6-27.7]$ & & $18.4[9.8-31.7]$ & \\
\hline aAOR $[95 \% \mathrm{CI}]^{\mathrm{d}}$ & 1.00 & $3.50[2.06-5.93]$ & $<0.001$ & $3.72[1.74-7.94]$ & 0.001 \\
\hline \multicolumn{6}{|l|}{ STI/HIV risk perception } \\
\hline \multicolumn{6}{|l|}{ Self-perceived STI risk } \\
\hline Not at all at risk & $73.6[72.2-74.9]$ & $31.5[23.7-40.7]$ & & $33.6[22.4-46.9]$ & \\
\hline Not very much & $23[21.7-24.3]$ & $51.1[42.3-59.8]$ & & $45.8[34.1-58.0]$ & \\
\hline Quite a lot & $2.6[2.2-3]$ & $11.9[7.1-19.5]$ & & $15.7[8.6-27.0]$ & \\
\hline Greatly at risk & $0.9[0.7-1.2]$ & $5.4[2.3-12.4]$ & & $4.9[1.7-13.4]$ & \\
\hline AOR $[95 \% \mathrm{Cl}]^{\mathrm{e}}$ & 1.00 & $5.60[3.28-9.56]$ & $<0.001$ & $7.04[3.69-13.43]$ & $<0.001$ \\
\hline \multicolumn{6}{|l|}{ Self-perceived HIV risk } \\
\hline Not at all at risk & $76.0[74.7-77.3]$ & $35.0[27.0-43.9]$ & & $39.2[28.2-51.5]$ & \\
\hline Not very much & $21.0[19.8-22.3]$ & $50.6[41.6-59.5]$ & & $44.5[33.0-56.7]$ & \\
\hline Quite a lot & $1.9[1.6-2.4]$ & $9.5[5.9-14.9]$ & & $10.9[6.0-19.1]$ & \\
\hline Greatly at risk & $1.1[0.8-1.4]$ & $4.9[2.1-11.2]$ & & $5.3[2.0-13.7]$ & \\
\hline $\mathrm{AOR}[95 \% \mathrm{Cl}]^{\mathrm{e}}$ & 1.00 & $5.44[3.26-9.08]$ & $<0.001$ & $6.42[3.67-11.23]$ & $<0.001$ \\
\hline \multicolumn{6}{|l|}{ Sexual health outcomes } \\
\hline Sexual health clinic attendance, past year & $14.1[12.5-16]$ & $33.0[24.8-42.4]$ & & $31.9[22.0-43.7]$ & \\
\hline $\mathrm{AOR}[95 \% \mathrm{Cl}]^{\mathrm{e}}$ & 1.00 & $4.71[2.58-8.58]$ & $<0.001$ & $4.53[2.30-8.94]$ & $<0.001$ \\
\hline Tested for HIV, past year & $3.4[2.9-4]$ & $17.0[12.0-23.4]$ & & $21.7[14.9-30.4]$ & \\
\hline AOR $[95 \% \mathrm{Cl}]^{\mathrm{e}}$ & 1.00 & 6.08 [3.92-9.41] & $<0.001$ & $9.44[5.59-15.96]$ & $<0.001$ \\
\hline STI diagnosis, past year & $0.9[0.7-1.2]$ & $4.9[2.6-9.2]$ & & $6.3[2.9-13.3]$ & \\
\hline AOR $[95 \% \mathrm{Cl}]^{\mathrm{e}}$ & 1.00 & $5.67[2.67-12.04]$ & $<0.001$ & $7.85[3.27-18.83]$ & $<0.001$ \\
\hline Lowest quintile of distribution of scores for Natsal-SF ${ }^{f}$ & $19.7[18.4-21.1]$ & $36.2[28.2-45.2]$ & & 28.0 [17.9-40.9] & \\
\hline AOR $[95 \% \mathrm{Cl}]^{\mathrm{e}}$ & 1.00 & $2.28[1.57-3.33]$ & $<0.001$ & $1.60[0.95-2.69]$ & 0.079 \\
\hline Feel distressed/worried about sex life & $10.2[9.3-11.2]$ & $14.1[9.0-21.2]$ & & $12.7[6.3-24.1]$ & \\
\hline $\mathrm{AOR}[95 \% \mathrm{Cl}]^{\mathrm{e}}$ & 1.00 & $1.39[0.85-2.27]$ & 0.194 & $1.14[0.57-2.29]$ & 0.703 \\
\hline Sought professional help/advice for sex life, past year & $7.1[6.3-7.9]$ & $16.5[11.0-24.0]$ & & $14.2[8.1-23.7]$ & \\
\hline $\mathrm{AOR}[95 \% \mathrm{Cl}]^{\mathrm{e}}$ & 1.00 & $2.70[1.65-4.42]$ & $<0.001$ & $3.04[1.62-5.68]$ & 0.001 \\
\hline
\end{tabular}


Table 5 Sexual behaviours, risk perception, and sexual health outcomes reported by men who have sex exclusively with women (MSEW), all men who have sex with men (MSM), and MSM who identified as gay in Natsal-3 (Continued)

\begin{tabular}{|c|c|c|c|c|c|}
\hline Experienced attempted non-volitional sex, ever & $4.3[3.7-5.0]$ & $22.8[16.0-31.4]$ & & $24.6[15.1-37.4]$ & \\
\hline AOR $\left[95 \% \mathrm{Cl}^{\mathrm{e}}\right.$ & 1.00 & $6.51[4.22-10.06]$ & $<0.001$ & $6.30[3.66-10.83]$ & $<0.001$ \\
\hline \multicolumn{6}{|c|}{ 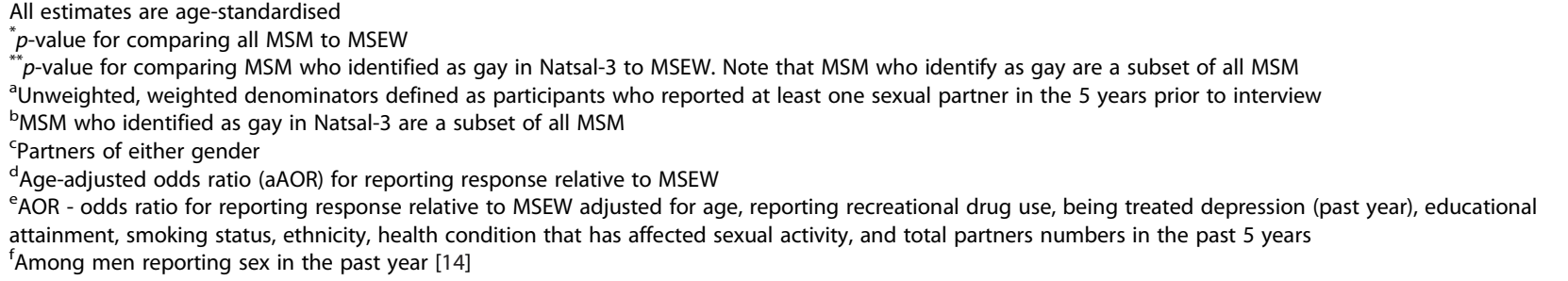 } \\
\hline
\end{tabular}

was often higher for gay-identifying MSM relative to all MSM (although confidence intervals overlap), and thus aAORs were larger, e.g., health status reported as 'bad' or 'very bad' (aAORs 2.23 vs. 1.67, respectively, relative to MSEW). The largest difference in aAORs between gay-identifying MSM and all MSM, relative to MSEW, was observed for reporting receiving treatment for depression in the past year (aAORs 4.03 vs. 2.75 , respectively, although confidence intervals again overlap). This suggestion of greater inequality for gay-identifying MSM was less evident in terms of sexual behaviours and outcomes. For example, the aAORs were smaller for gay-identifying MSM than for all MSM for reporting condomless sex and concurrency. For the sexual health outcomes studied, the differences with MSEW were similar, except that gay-identifying MSM were as likely as MSEW to have poor sexual function, while the aAOR for poor sexual function was significantly greater for all MSM relative to MSEW. In summary, and as evident from Tables 6 and 7, these findings suggest marginally greater clustering of poor health outcomes (but not poor health behaviours) for gay-identifying MSM than for all MSM, and in turn, for MSEW.

\section{Discussion}

National probability survey data show that only a small minority of men in Britain are MSM in terms of reporting sex with men in the past five years, and of these men, around half identify as gay. While many of the harmful health behaviours and poor health outcomes studied were reported by a minority of MSM, this was often a larger proportion than observed among MSEW, and they were also more likely to be reported in combination and across health domains, demonstrating the considerable health inequality that exists for MSM in Britain, often regardless of whether or not MSM identify as gay.

A major strength of this study is that it uses data from a large national probability survey such that the findings can be considered as broadly representative of the British general population [11]. In addition, unlike the publication of data on MSM collected by Natsal-2 [19], this study has exploited the uniqueness of the Natsal data in terms of them being collected from all men, by considering the well-being of MSM relative to MSEW. The ability to sample from the large proportion of MSM who did not identify as gay is a further strength, and shows that a broader group of MSM are represented in this study than in previous studies, which have tended to sample from the gay scene in clubs or sexual health clinic attendees, and so have reported greater sexual risk and drug use behaviours and adverse sexual health outcomes $[8-10,20]$. However, limitations are that the population prevalence of MSM results in a relatively small sample of MSM in Natsal, with insufficient statistical power to stratify MSM according to their sexual identity. While we presented estimates specifically for gay-identifying MSM alongside those for all MSM, the size of this sub-sample means that their estimates should be interpreted with caution. Including men who reported ever having had same-sex sex would have increased the number of MSM in our sample, but as some men may have had a transient experience we chose to focus on the past five years to minimise this effect, and also to present a contemporary picture of MSM in Britain. Some misclassification bias is also likely with our comparator group of MSEW as, by definition, this includes men who have not yet had, but who go on to have, male partners in the future, as well as MSEW who reported same-sex sex more than five years ago. However, the latter corresponds to a very small minority of MSEW (2.5\%), and so the in/exclusion of these 115 men has little impact on the magnitude of the estimates given the large number of MSEW in Natsal. Finally, as with all survey data we rely on what participants report so our data are subject to reporting and social desirability bias, and as a cross-sectional survey we are unable to assume causality.

An important strength of this paper is that it considers sexual health beyond STI/HIV transmission risk. By including a psychometrically-validated measure of sexual function, the Natsal-SF [14], and data about everexperience of non-volitional sex, mental and physical 
Table 6 The extent that harmful behaviours ${ }^{a}$ cluster within and across health domains for (i) men reporting sex exclusively with women (MSEW), (ii) men reporting sex with men (MSM), and (iii) and MSM who identified as gay in Natsal-3

\begin{tabular}{lll}
$\begin{array}{l}\text { Population: } \\
\text { Clustering: } \\
\text { Domain: }\end{array}$ & \multicolumn{1}{c}{ Within domain } \\
& $\begin{array}{l}\text { Physical health } \\
\%(95 \% \mathrm{Cl})\end{array}$ & $\begin{array}{l}\text { Sexual health } \\
\%(95 \% \mathrm{Cl})\end{array}$ \\
$\begin{array}{l}\text { No. of harmful behaviours } \\
\text { reported within each domain: }\end{array}$ & & \\
0 & $54.5(52.8-56.1)$ & $86.3(85.3-87.3)$ \\
1 & $24.6(23.3-26.1)$ & $8.7(7.9-9.6)$ \\
2 & $14.8(13.7-15.9)$ & $4.7(4.1-5.3)$ \\
3 & $5.0(4.4-5.7)$ & $0.4(0.2-0.5)$ \\
4 & $1.2(0.9-1.5)$ & N/A \\
& Total: $100 \%$ & Total: $100 \%$
\end{tabular}

Unweighted=4641 weighted:5727

Population:

Clustering:

Domain:

No. of harmful behaviours reported within each domain:

0
1
2
3
4

Population:

Clustering:

Domain:

No. of harmful behaviours reported within each domain:$$
0
$$$$
1
$$$$
2
$$$$
3
$$$$
4
$$

Within domain

Physical health

$\%(95 \% \mathrm{Cl})$

$34.5(26.7-43.1)$
$36.4(28.0-45.7)$
$16.4(11.5-22.9)$
$8.9(4.9-15.7)$
$3.8(2.0-7.3)$
Total: $100 \%$

Total: $100 \%$

(ii) Men who have sex with men (MSM)

Across domains

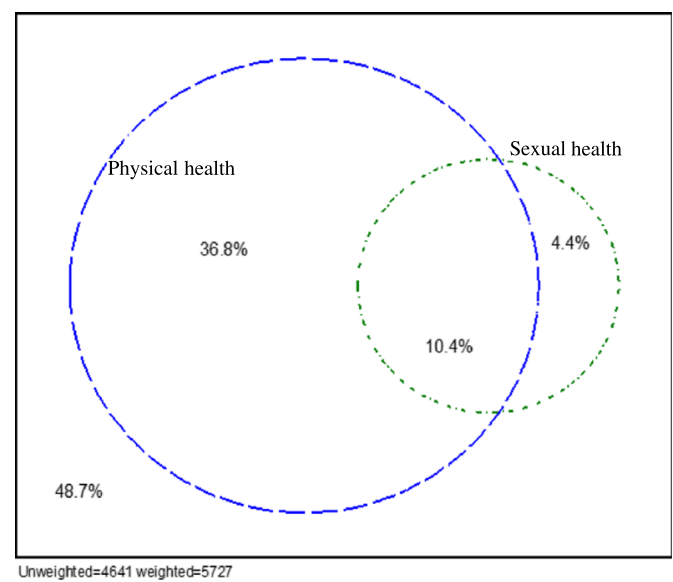

Across domains

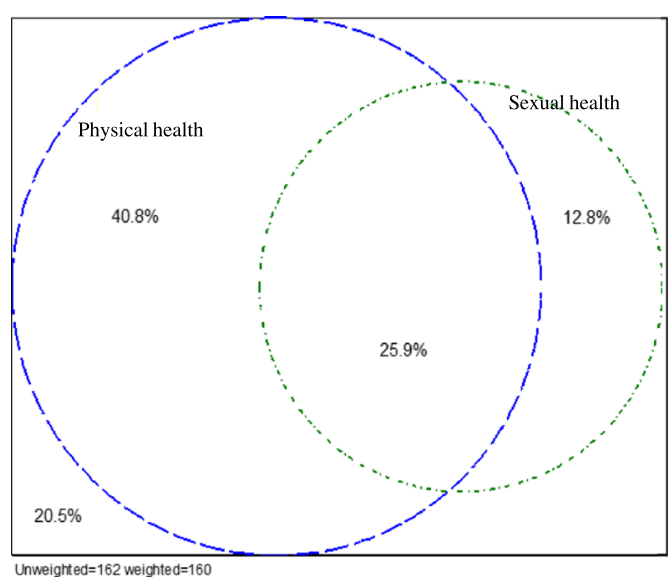

(iii) MSM who identified as gay ${ }^{b}$

Within domain

Physical health Sexual health

$\%(95 \% \mathrm{Cl}) \quad \%(95 \% \mathrm{Cl})$

$\begin{array}{ll}34.1(24.7-44.9) & 75.7(61.7-85.7) \\ 40.9(29.5-53.4) & 12.7(5.7-25.9) \\ 16.4(10.0-25.8) & 10.6(4.3-23.9) \\ 4.0(1.4-11.1) & 1.1(0.3-4.5) \\ 4.7(2.2-9.9) & \text { N/A } \\ \text { Total: } 100 \% & \text { Total: } 100 \%\end{array}$

Across domains

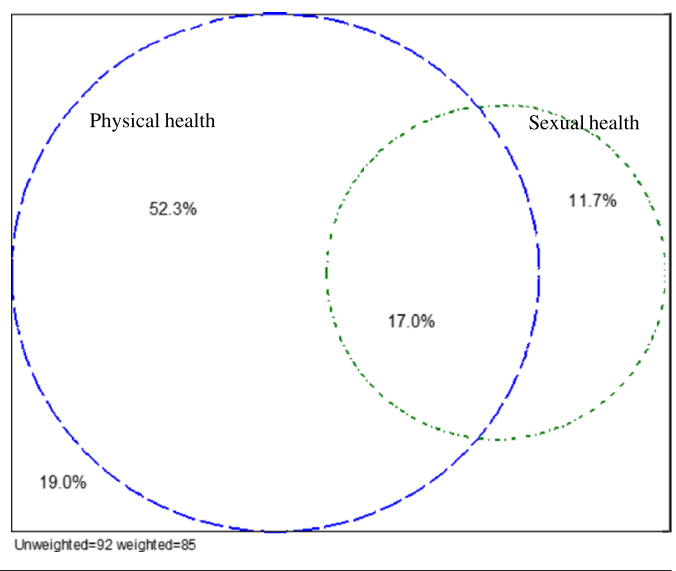


Table 7 The extent that poor health outcomes ${ }^{a}$ cluster within and across health domains for (i) men reporting sex exclusively with women (MSEW), (ii) men reporting sex with men (MSM), and (iii) and MSM who identified as gay in Natsal-3

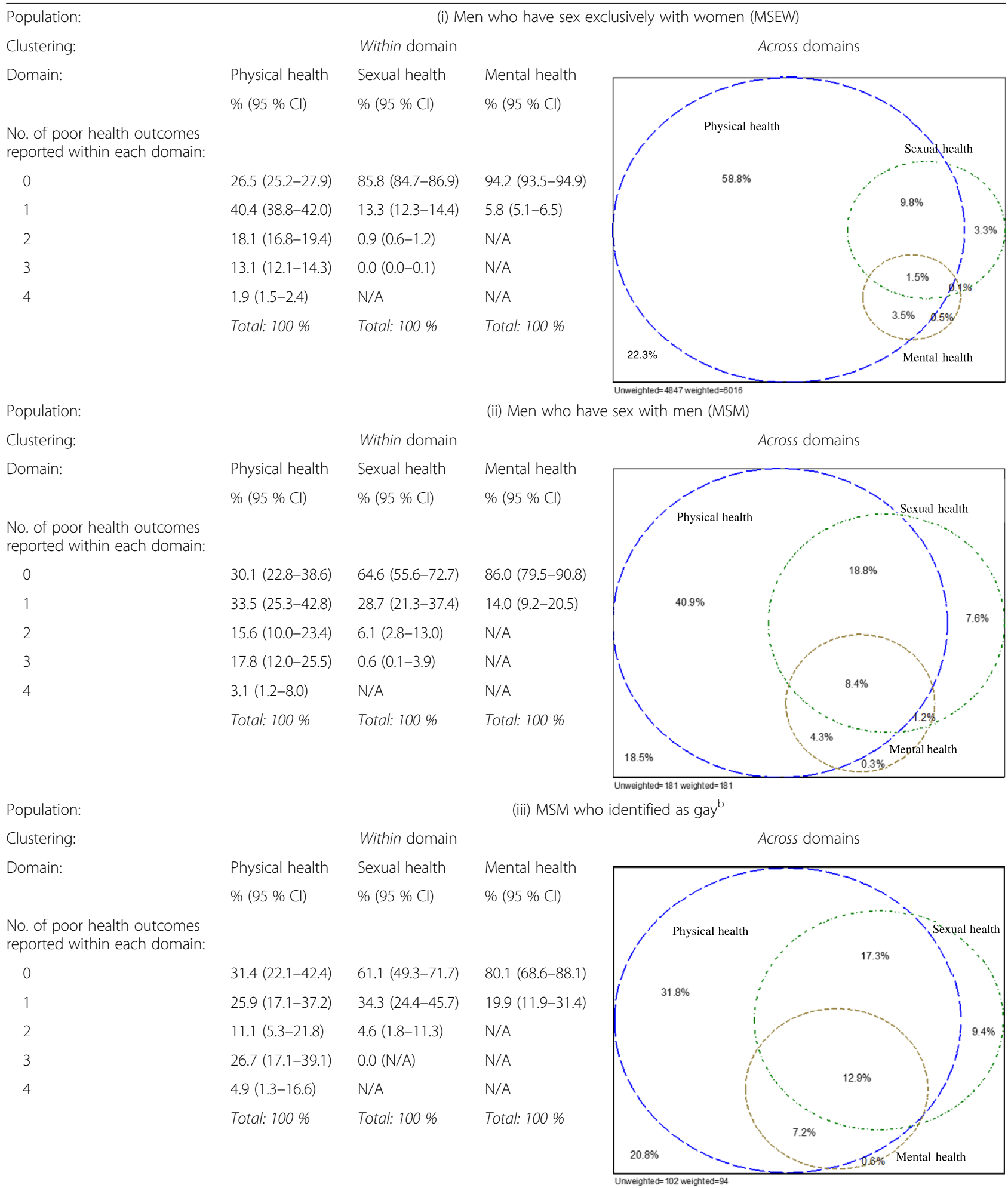


health status and behaviours considered detrimental to health, we have been able to consider the wider health and well-being needs of MSM. Our endeavour is consistent with the rationale for the broader framing of sexual health [3], and Public Health England's recent action plan for promoting the health and wellbeing of gay, bisexual and other MSM by taking into account their wider health issues and inequalities and the broader socio-economic and cultural context. [5] We recognise that the variables used to define the health domains we focused on are not exhaustive, reflecting the available data. Of note, Natsal-3 was relatively limited in capturing mental health, due to the complexity of doing so within a questionnaire with primarily a sexual health focus and the relatively low prevalence of conditions other than depression [4]. In this respect, we chose to focus on reported treatment for depression as we sought to identify individuals experiencing diagnosed depression at the more severe end of the clinical spectrum [4].

Natsal's rich sexual behaviour data enabled us to examine the number of male and female sexual partners reported in various timeframes, including new partners and condomless sex partners. MSM reported larger numbers of partners on average compared to MSEW, as reported elsewhere [21]. However, the bimodal distribution of partner numbers we observed for MSM, with a small but non-negligible proportion of MSM reporting very few partners, challenges stereotypes inferred from clinical data and community surveys of gay men [8-10]. Additionally, considerably larger proportions of MSM reported female partners than observed in convenience surveys [9, 10], and as similar proportions of MSM and MSEW reported having new female partners in the past year, then our data support the notion that some MSM may act as a bridge population for STI/HIV transmission between higher-risk and lower-risk sexual networks [22].

Having larger numbers of sexual partners, and particularly multiple partners without using condoms, are both recognised as associated with a number of adverse sexual health outcomes, including STI/HIV acquisition [23], and both were more commonly reported by MSM than MSEW. Perhaps consequentially, MSM were more likely than MSEW to perceive themselves as at risk of STI/ HIV acquisition, although the majority considered their risk as 'not very much'. This may, in part, explain our finding that the majority of MSM reported that they had not been to a sexual health clinic and/or tested for HIV in the past year; figures that are far lower than observed from clinical audit $[8,24]$, suggested by convenience surveys $[9,10]$, or recommended by national guidelines $[25,26]$. MSM were on the other hand more likely than MSEW to report seeking help/advice regarding their sex life (particularly from sexual health clinics) and to have taken drugs to assist their sexual performance. While this may suggest that MSM are better at seeking help for their sex lives than MSEW, it may reflect that MSM were more likely than MSEW to have poorer overall sexual function according to the validated Natsal-SF measure [14]. Although this hypothesis is consistent with findings from large surveys of gay men undertaken in the US and Australia that observed very high reported prevalence of sexual function problems $[27,28]$, it is worth noting that the difference in sexual function was smaller when the analysis was limited to gay-identifying MSM. Our finding of a higher prevalence of ever experiencing attempted non-volitional sex among MSM (regardless of whether they identified as gay) than among MSEW also supports other studies [29, 30].

As others have previously reported [6], we observed higher levels of poor mental health among MSM in contrast to MSEW, at least in terms of the proportion reporting receiving treatment for depression [31]. In addition, MSM reported greater substance use in terms of smoking and recreational drug use. However, to reiterate, many of the harmful health behaviours studied were often reported by only a minority of MSM, and considerably smaller proportions than observed from clinic and community surveys of MSM [8-10]. Furthermore, MSM and MSEW were often similar in terms of their physical health, contradicting previous research [32], although when we focused on gay-identifying MSM, these differences with MSEW were often larger. Health inequalities were also evident in the domain of sexual health with MSM more likely than MSEW to report both harmful sexual behaviours and poor sexual health outcomes, to report these in combination, and in conjunction with those in other health domains.

\section{Conclusions}

These data suggest that there is a small but non-negligible group of MSM - and not necessarily MSM who identify as gay - who are likely to benefit from interventions that adopt a more holistic approach to improving health and wellbeing. However, these should not be at the expense of interventions that seek to tackle the ongoing STI/HIV epidemic among the MSM population [1, 2]. Policies and interventions that promote regular testing, condom use, and safe sexual behaviour among MSM at risk of infection [24-26] should therefore remain a priority in order to improve the health of individuals as well as public health. Achieving this in practice requires a two-pronged approach: Sexual health clinics need to offer MSM more holistic care, either inhouse or through referral, while healthcare professionals in general medical services require a greater awareness of MSM (for example, by routinely asking about sexual orientation as the NHS recommends [33] as well as the gender of their sexual partners), and that their healthcare includes, but is not limited to the sexual health needs of MSM. 
While this paper has sought to advance the literature by considering health inequalities beyond STIs and HIV, and has presented evidence of the extent to which health inequalities cluster for some MSM, future research needs to understand the mechanisms underlying these inequalities, how and why they cluster. Adopting an even broader perspective that goes beyond the proximal determinants of health, by seeking insight into distal determinants, for example in terms of the sociocultural context, will be helpful for gaining this understanding and for developing interventions that can effectively address multifaceted inequalities.

\begin{abstract}
Abbreviations
AOR, adjusted odds ratio; BMI, body mass index; CAPI, computer-assisted personal-interview; CASI, computer-assisted self-interview; HIV, human immunodeficiency virus; IMD, Index of Multiple Deprivation; MSEW, men who have with women; MSM, men who have sex with men; Natsal, National Survey of Sexual Attitudes and Lifestyles; NS-SEC, National Statistics SocioEconomic Classification; STI, sexually transmitted infection
\end{abstract}

\section{Acknowledgements}

Natsal-3 is a collaboration between University College London, the London School of Hygiene \& Tropical Medicine, NatCen Social Research, Public Health England (formerly the Health Protection Agency), and the University of Manchester. We thank the study participants, the team of interviewers from NatCen Social Research who carried out the interviews, and operations and computing staff from NatCen Social Research.

\section{Funding}

The study was supported by grants from the Medical Research Council [G0701757]; and the Wellcome Trust [084840]; with contributions from the Economic and Social Research Council and Department of Health. NF is supported by a NIHR Academic Clinical Lectureship.

\section{Role of the funding source}

The funders of the study had no role in study design, data collection, data analysis, data interpretation, or writing of the report. The corresponding author had full access to all the data in the study and had final responsibility for the decision to submit for publication.

\section{Availability of data and materials}

The dataset supporting the conclusions of this article is available in the UK Data Service repository, unique persistent identifier: 10.5255/UKDA-SN-7799-1; https://discover.ukdataservice.ac.uk/catalogue/?sn=7799\&type=Data\%20 catalogue.

\section{Authors' contributions}

The paper was conceived by CHM, NF, AMJ, PS, CT, GH and AN. CHM wrote the first draft, with further contributions from all authors. Statistical analysis was done by PP with support from CHM. CHM, PS, KW, WM, and AMJ (Principal Investigator), initial applicants on Natsal-3, wrote the study protocol and obtained funding. Natsal-3 questionnaire design, ethics applications, and piloting were undertaken by SC, NF, CT, PS, and AMJ. Data management was undertaken by NatCen Social Research (SC) and UCL (CHM, CT, NF). All authors contributed to data interpretation, reviewed successive drafts, and approved the final version of the manuscript.

\section{Competing interests}

AMJ has been a Governor of the Wellcome Trust since 2011. The other authors declare that they have no competing interests.

\section{Author details}

${ }^{1}$ Research Department of Infection and Population Health, University College London, 3rd floor Mortimer Market Centre, London, UK. ${ }^{2}$ Department of Social and Environmental Health Research, London School of Hygiene and Tropical Medicine, Tavistock Place, London, UK. ${ }^{3}$ Public Health England, Colindale, London, UK.
Received: 15 January 2016 Accepted: 25 May 2016

Published online: 07 July 2016

\section{References}

1. Yin Z, Brown AE, Hughes G, Nardone A, Gill ON, Delpech V. HIV in the United Kingdom 2014 Report: Data to end 2013. London: Public Health England; 2014.

2. Public Health England. Sexually transmitted infections and chlamydia screening in England, 2013. HPR. 2015:9:22.

3. WHO. Defining sexual health: report of a technical consultation on sexual health, 28-31 January 2002, Geneva. 2006. http://www.who.int/ reproductivehealth/publications/sexual_health/defining_sh/en/. (Accessed 26 Nov 2015).

4. Field $\mathrm{N}$, Mercer $\mathrm{CH}$, Sonnenberg $\mathrm{P}$, et al. Associations between health and sexual lifestyles in Britain: findings from the third National Survey of Sexual Attitudes and Lifestyles (Natsal-3). Lancet. 2013;382:1830-44.

5. Public Health England. Promoting the health and wellbeing of gay, bisexual and other men who have sex with men. London. 2014. https://www.gov. uk/government/publications/promoting-the-health-and-wellbeing-of-gaybisexual-and-other-men-who-have-sex-with-men. (Accessed 26 Nov 2015).

6. King M, Semlyen J, See Tai S, et al. A systematic review of mental disorder, suicide and deliberate self harm in lesbian, gay and bisexual people. BMC Psychiatry. 2008;18:70.

7. Vosburgh HW, Mansergh G, Sullivan PS, Purcell DW. A review of the literature on event-level substance use and sexual risk behavior among men who have sex with men. AIDS Behavior. 2012:16:1394-410.

8. Desai M, Desai S, Sullivan AK, et al. Audit of HIV testing frequency and behavioural interventions for men who have sex with men: policy and practice in sexual health clinics in England. Sex Transm Infect. 2013;89:404-8.

9. Reid D. Vital Statistics 2010: The UK Gay Men's Sex Survey Data Report: All England by Strategic Health Authority of Residence. London: Sigma Research; 2011.

10. Schwarcz S, Spindler H, Scheer S, Valleroy L, Lansky A. Assessing representativeness of sampling methods for reaching men who have sex with men: a direct comparison of results obtained from convenience and probability samples. AIDS Behav. 2007;11:596-602.

11. Erens B, Phelps A, Clifton S, et al. Methodology of the third British National Survey of Sexual Attitudes and Lifestyles (Natsal-3). Sex Transm Infect. 2013. doi:10.1136/sextrans-2013-051359.

12. Johnson AM, Mercer $\mathrm{CH}$, Erens $\mathrm{B}$, et al. Sexual behaviour in Britain: partnerships, practices, and HIV risk behaviours. Lancet. 2001;358:1835-42.

13. Ruf M, Delpech V, Osuagwu U, Brown AE, Robinson E, Chadborn T. Men who have sex with men: estimating the size of at-risk populations in London primary care trusts. Int J STD AIDS. 2011;22(1):25-9.

14. Mitchell KR, Ploubidis GB, Datta J, Wellings K. The Natsal-SF: a validated measure of sexual function for use in community surveys. Eur J Epidemiol. 2012;27:409-18

15. Arroll B. Screening for depression in primary care with two verbally asked questions: cross sectional study. BMJ. 2003;327:1144-6.

16. Arroll B, Goodyear-Smith F, Crengle S, et al. Validation of PHQ-2 and PHQ-9 to screen for major depression in the primary care population. Ann Fam Med. 2010;8:348-53.

17. Office for National Statistics. Standard Occupational Classification 2010, vol. 1-3. Basingstoke: Palgrave Macmillan; 2010.

18. Payne RA, Abel GA. UK indices of multiple deprivation-a way to make comparisons across constituent countries easier. Health Stat Q. 2012;53:22-37.

19. Mercer $\mathrm{CH}$, Fenton KA, Copas AJ, et al. Increasing prevalence of male homosexual partnerships and practices in Britain 1990-2000: Evidence from national probability surveys. AIDS. 2004:18:1453-8.

20. Prah P, Hickson F, Bonell C, et al. Men who have sex with men in Great Britain: comparing methods and estimates from probability and convenience sample surveys. Sex Transm Infect. 2016. doi:10.1136/sextrans2015-052389.

21. Centers for Disease Control and Prevention. HIV risk, prevention, and testing behaviors - National HIV behavioral surveillance system: Men who have sex with men, 20 U.S. cities, 2011. HIV Surveillance Special Report 8. 2014. http://www.cdc.gov/hiv/library/reports/surveillance/\#special. (Accessed 20 Apr 2016).

22. Gorbach PM, Murphy R, Weiss RE, Hucks-Oritz C, Shoptaw S. Bridging sexual boundaries: Men who have sex with men and women in a street-based sample in Los Angeles. J Urban Health. 2009;86(S1):63-76. 
23. Sonnenberg P, Clifton S, Beddows S, et al. Prevalence, risk factors, and uptake of interventions for sexually transmitted infections in Britain: findings from the National Surveys of Sexual Attitudes and Lifestyles (Natsal). Lancet. 2013:382:1795-806.

24. McClean H, Sullivan AK, Carne CA, et al. UK national audit against the key performance indicators in the British Association for Sexual Health and HIV Medical Foundation for AIDS and Sexual Health Sexually Transmitted Infections Management Standards. Int J STD AIDS. 2012;23:742-7.

25. National Institute for Health and Clinical Excellence. Increasing the Uptake of HIV Testing Among men who Have sex With men. London: NICE; 2011.

26. BASHH. Recommendations for Testing for Sexually Transmitted Infections in men who Have sex With men. London: BASHH; 2014

27. Hirshfield S, Chiasson MA, Wagmiller Jr RL, et al. Sexual dysfunction in an Internet sample of U.S. men who have sex with men. J Sex Med. 2010;7:3104-14

28. Mao L, Newman CE, Kidd MR, Saltman DC, Rogers GD, Kippax SC. Self-reported sexual difficulties and their association with depression and other factors among gay men attending high HIV-caseload general practices in Australia. J Sex Med. 2009;6:1378-85.

29. Coxell A, King M, Mezey G, Gordon D. Lifetime prevalence, characteristics, and associated problems of non-consensual sex in men: cross sectional survey. BMJ. 1999;318:846-50

30. Walters M, Chen J, Breiding M. The National Intimate Partner and Sexual Violence Survey (NISVS): 2010 Findings on Victimization by Sexual Orientation. Atlanta: Centers for Disease Control and Prevention; 2013.

31. Field N, Prah P, Mercer $\mathrm{CH}$, et al. Are depression and poor sexual health neglected co-morbidities? Evidence from a population sample. BMJ Open. 2016. doi:10.1136/bmjopen-2015-010521.

32. Lick DJ, Durson LE, Johnson KL. Minority stress and physical health among sexual minorities. Perspect Psychol Sci. 2013;8:521-48.

33. NHS England/Equality and Health Inequalities Team. Monitoring equalities and health inequalities: A position paper. 2015. https://www.england.nhs.uk/wpcontent/uploads/2015/03/monitrg-ehi-pos-paper.pdf. (Accessed 26 Nov 2015).

34. NHS Choices website. Binge drinking. The Information Standard member organisation. http://www.nhs.uk/Livewell/alcohol/Pages/Bingedrinking.aspx. (Accessed 20 Apr 2016).

35. National Obesity Observatory. Body Mass Index as a Measure of Obesity. London: Public Health England; 2009.

\section{Submit your next manuscript to BioMed Central and we will help you at every step:}

- We accept pre-submission inquiries

- Our selector tool helps you to find the most relevant journal

- We provide round the clock customer support

- Convenient online submission

- Thorough peer review

- Inclusion in PubMed and all major indexing services

- Maximum visibility for your research

Submit your manuscript at www.biomedcentral.com/submit

) Biomed Central 